\title{
Network Innovators Community Event (GENI NICE 2016)
}

\author{
Mark Berman \\ Raytheon BBN Technologies \\ 10 Moulton Street \\ Cambridge, MA 02138 \\ +1 (617) 873-3675 \\ mberman@bbn.com
}

\author{
Ibrahim Matta \\ Boston University \\ 111 Cummington Mall \\ Boston, MA 02215 \\ +1 (617) 358-1062 \\ matta@bu.edu
}

\author{
Violet R. Syrotiuk \\ Arizona State University \\ P.O. Box 878809 \\ Tempe, AZ 85287 \\ +1 (480) 965-7034 \\ syrotiuk@asu.edu
}

\author{
Vicraj Thomas \\ Raytheon BBN Technologies \\ Park Place East, Ste. 630 \\ 5775 Wayzata Blvd. \\ St. Louis Park, MN 55416 \\ +1 (952) 545-5713 \\ vthomas@bbn.com
}

\begin{abstract}
The Network Innovators Community Event (GENI NICE) is the premier event for researchers to demonstrate and present research results and to discuss work in progress related to the NSF's Global Environment for Network Innovations (GENI) and related advanced cyberinfrastructure testbeds.

Growing out of the strong experiment- and demonstration-driven tradition of the GENI Engineering Conferences (GECs), NICE specializes in direct experimenter-to-experimenter interaction and information exchange. NICE 2016 represents the second year of NICE, and its first year being held in conjunction with CoNEXT.
\end{abstract}

\section{Keywords}

GENI; Future Internet; Next generation networking; Future Internet testbeds; Distributed cloud computing, Edge cloud, Edge computing; Cellular research networks; Software-defined exchanges; Software-defined infrastructure.

\section{The NICE Community}

The Network Innovators Community Event (GENI NICE) provides a venue for a free and rapid exchange of information among researchers, educators, system builders, and IT professionals who make, sustain, and use advanced research cyberinfrastructure testbed environments. These include the Global Environment for Network Innovations (GENI), as well as related future Internet and distributed cloud (FIDC) testbeds and research infrastructures.

NICE brings together multiple interconnected segments of this dynamic and growing community. In an environment of shared, distributed cyberinfrastructure, effective and collaborative interaction among these groups is vital to sustaining rapid research progress.

End users: Researchers and educators who use advanced research cyberinfrastructure testbeds in their work.

Testbed builders: Systems researchers and builders who design and develop new research cyberinfrastructure concepts.

Infrastructure owners and operators: Chief information officers (CIOs) and information technology professionals who own and operate research cyberinfrastructure on behalf of end users.

Permission to make digital or hard copies of part or all of this work for personal or classroom use is granted without fee provided that copies are not made or distributed for profit or commercial advantage and that copies bear this notice and the full citation on the first page. Copyrights for third-party components of this work must be honored. For all other uses, contact the Owner/Author. Copyright is held by the owner/author(s).

CoNEXT'16, December 12-15, 2016, Irvine, CA, USA

ACM 978-1-4503-4292-6/16/12.

DOI: http://dx.doi.org/10.1145/2999572.3004860

\section{The NICE Agenda}

This year's event, NICE 2016, takes place at a particularly exciting time for this community. As the GENI project begins its transition to a broader management paradigm, a new, researchdriven consortium is forming to play a key role both in GENI oversight and in driving the vision of new cyberinfrastructure research.

This is also a time of great potential for new developments in advanced research cyberinfrastructure testbeds, as researchers and sponsors are increasingly converging on key areas to pursue. As an example, the recent "Looking Beyond the Internet" report [1] identifies several promising areas for research testbed investment. One of these areas, city-scale wireless research testbeds, is addressed in a recently announced White House initiative, spearheaded by the NSF.

The NICE community is ideally positioned both to provide input to and to derive benefit from these important developments, and this year's event agenda draws heavily on these topics.

Sessions for NICE 2016 include the following.

Opening Session: In addition to the keynote presentation, this session includes an update on the Future Cyberinfrastructure Consortium, including the consortium's mission and goals, as well as how the community can participate in the consortium.

Session chair: Mark Berman (GENI project office).

Software-Defined Exchanges: This session explores recent developments and upcoming research in software-defined exchanges (SDXs) and software-defined infrastructure (SDI). These interrelated approaches apply software-defined networking concepts and technology to facilitate the sharing and exchange of networking and heterogeneous cyberinfrastructure resources.

Session chairs: Russ Clark (Georgia Institute of Technology) and Tom Lehman (University of Maryland).

Non-IP Experiments: One of the motivations of Future Internet and Distributed Cloud (FIDC) testbeds is the ability to conduct experiments using network protocols that are not compatible with the standard Internet protocol (IP) or transmission control protocol (TCP). This session reports on experiments in future Internet architectures and other research that explore this research space.

Session chairs: Nirmala Shenoy (Rochester Institute of Technology) and Ibrahim Matta (Boston University).

Wireless Experiments: This session addresses recent application work at campuses using 4G LTE technology. Areas addressed 
include heterogeneous vehicular networking, cloud-based radio processing and voice over LTE (VO-LTE) for public safety.

Session chairs: Abhimanyu Gosain (GENI project office) and Ivan Seskar (Rutgers University).

Joint Panel Session "Picking the Right Testbed for Your Experiment": This joint panel brings together participants from the NICE community as well as the co-located Cloud-Assisted Networking (CAN) workshop and CoNEXT student workshop to discuss the relative benefits of different advanced cyberinfrastructure research testbeds and comparable commercial offerings.

Session chairs: Mike Zink (University of Massachusetts, Amherst) and Murat Yuksel (University of Central Florida).

Evening demo session: The demo session is a longstanding tradition extended from the GECs to NICE. It provides an informal opportunity for information exchange in a style that matches the experiment- and demonstration-driven culture of the NICE community. (See Figure 1.)

\section{ACKNOWLEDGMENTS}

GENI and NICE are supported by the National Science Foundation and the GENI Project Office. Any opinions, findings, conclusions or recommendations expressed in this material are the authors' and do not necessarily reflect the views of the NSF.

Our thanks to the organizers of CoNEXT 2016 for welcoming NICE to the CoNEXT community.

\section{REFERENCES}

[1] NSF CISE Looking Beyond the Internet Steering Group. 2016. "Final Report: Looking Beyond the Internet."

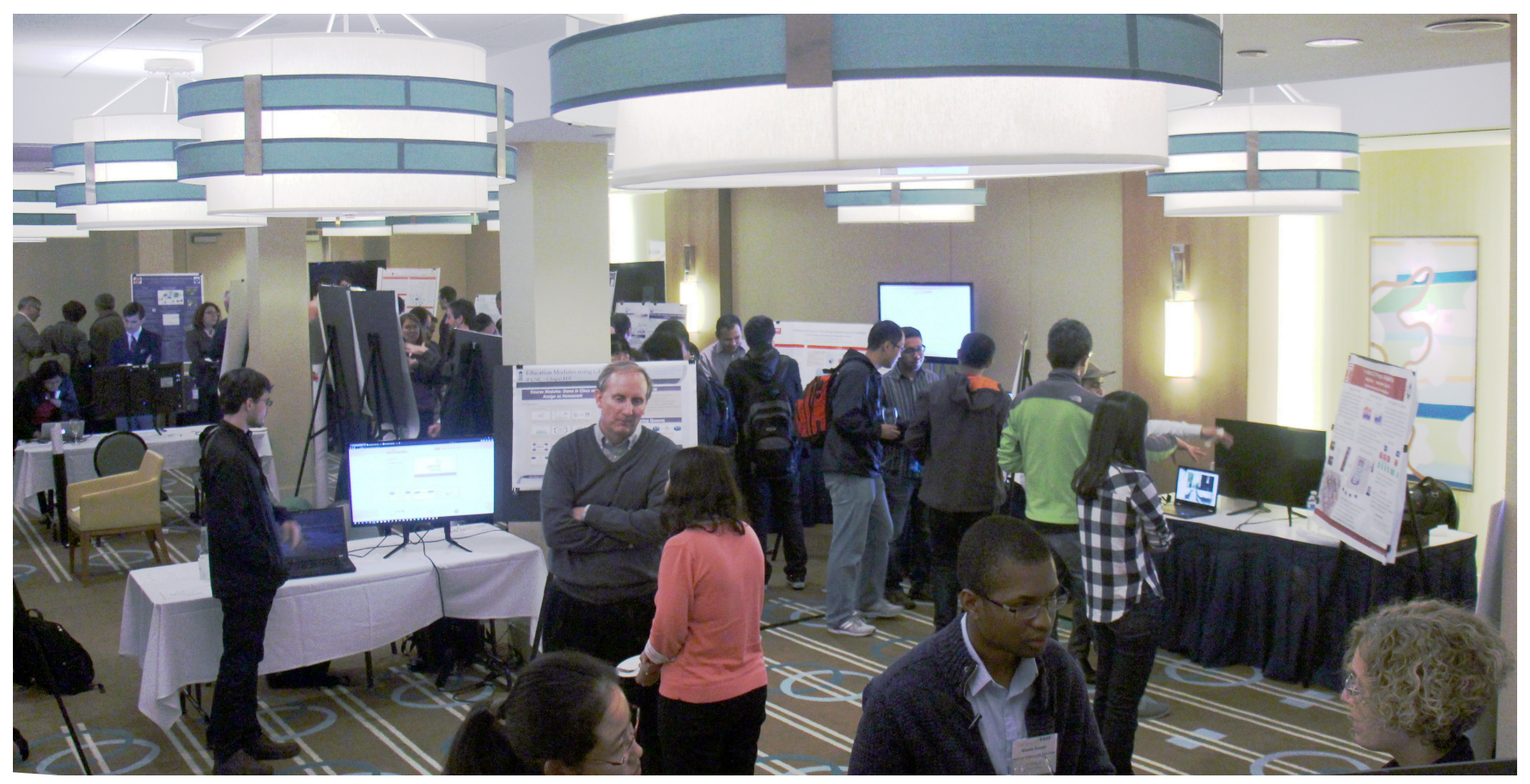

Figure 1: Demo Session at NICE 2015 